\title{
Methods of Popularizing Astronomy in Various Countries
}

\section{Cecylia Iwaniszewska}

Institute of Astronomy, N. Copernicus University, PL-87-100 Torun, Poland

\begin{abstract}
Some interesting methods of popularizing astronomy in various countries were reviewed, and may be divided into two types: active and passive methods. Active methods include:

1. Astronomy in the countryside. Astronomical camps for both children and grownups, who normally live in towns, to learn basic astronomical facts. (Italy)

2. Astro-puppets. Visitors to an observatory are greeted by puppets (Copernicus, Galileo, etc.) who not only give talks, but also converse with the audience. (Argentina)

3. Hand-operated devices. A simple orrery showing the movement of the Earth, and other devices that viewers operate for themselves. (India)

4. Graphical calendars. Several popular observatories collaborate to produce a yearly calendar showing planetary rising and setting times, etc. (Czechoslovakia)

5. Amateur clubs. High-school and university students became so interested in astronomy that they formed their own group and now produce a magazine and carry out observing, etc. in a country where there is no professional astronomical institution. (Paraguay)

6. An interdisciplinary approach. Various meetings and workshops are arranged to bring together astronomy, physics, biology, geology, etc., leading to a better understanding of modern science. (Japan)

7. Astronomical competitions. Everything from children's drawings to special tests and papers, sometimes connected with special events. Also awards for the best work of popularization in a given year. (Worldwide)

Passive methods are far more traditional, and include planetarium shows, including special ones for pilgrims (India); special exhibitions; radio and television programmes; public lectures; popular magazines usually edited for special groups of readers. Finally, what about humorous astronomical stories or pictures, is that active or passive?
\end{abstract}

